\title{
Klinische Ethik: Von der Bedeutung des autoritätsfreien Umgangs
}

\author{
Jean Martin
}

Ehemaliges Mitglied der nationalen Ethikkommission, Mitglied der Redaktion

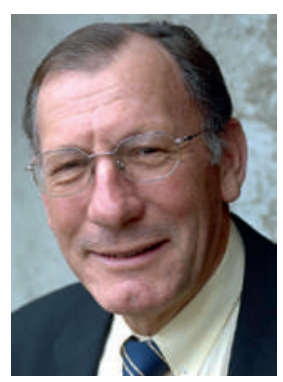

NB: Der Artikel bezieht sich auf die Situation in den USA, wo Ethikberater häufig Nichtmediziner sind. Dennoch betrifft die Argumentation alle, die in der klinischen Ethik tätig sind (auch in unserem Lan wird anerkannt, dass Ethik kein medizinisches

«Monopol» sein kann/darf).

\section{Fiester A. Teaching Non- authoritarian Clinical Ethics: Using an Inventory of Bioethical Positions. Hastings Center Report. 2015; 45(2):20-6.}

«Im nationalen Dialog zur Ausbildung und Zertifizierung klinischer Ethikberater (clinical ethics consultants) wird generell gefordert, deren jeweilige Kenntnisse und Kompetenzen zu verbessern. Es besteht weitgehend Konsens darüber, dass viele von ihnen nicht genügend ausgebildet sind.» So beginnt ein von der Leiterin des Mediationsprogramms für klinische Ethik der Universität Pennsylvania [1] veröffentlichter Artikel im Hastings Center Report. Und darin heisst es weiter: «Es besteht Einigkeit darüber, dass «autoritäre` Haltungen in der ethischen Beratung zu vermeiden sind, also ein Vorgehen, bei dem der Berater in komplexen oder konfliktbehafteten Situationen den betroffenen Personen seine eigenen ethischen Grundsätze oder religiösen Überzeugungen aufdrängt.»

Bislang - so Fiester - ist jedoch nur wenig geschehen, um klinische Ethiker und Ethikerinnen so zu schulen, dass sie nicht ihre eigenen Werte voranstellen. «Dazu muss zunächst einmal identifiziert werden, was denn diese persönlichen Überzeugungen bzw. normativen Vorstellungen überhaupt ausmacht, in einem Kontext vielfältigster, an sich zwar legitimer, aber gegensätzlicher Positionen, in einem pluralistisch geprägten Land wie den USA. Zur Einschätzung der eigenen Position im Vergleich zu anderen legitimen Standpunkten bedarf es der Distanz zu moralischen Sichtweisen, die man persönlich als absolut oder universell gültig sehen mag» (die dies jedoch nicht sind für andere). Es erscheint klar, dass für die Länder Europas, die auf einem vergleichbaren philosophischen und rechtlichen Fundament ruhen, gleiche Warnungen gelten.

Der Fiester-Artikel spricht von einem «Bioethical Positions Inventory", das fünfzehn Affirmationspaare zu ethischen Fragen beinhaltet (jedes Paar mit einer «konservativen» und einer «liberalen» Haltung). Die in Ausbildung befindlichen Ethikberater diagnostizieren zunächst ihre eigene Position und erarbeiten dann in einem zweiten Schritt eine Argumentation, mit der sie die jeweils entgegengesetzte Position verteidigen! (Diese Übung schützt davor, in fragwürdige «Wertehegemonien» zu verfallen - Fiester.) Parallel dazu müssen sie wie folgt eigene Erfahrungen diskutieren: 1. Beschreiben Sie eine Beratung, bei der die von Ihnen präferierte Position in direkter Opposition zu der des Patienten, der Familie oder des Therapeuten war. Wie haben Sie das Gespräch geführt? 2. Beschreiben sie einen Fall, wo Sie besorgt feststellen mussten, dass Sie die Bera- tung beeinflusst haben, damit das Ergebnis mit Ihren Werten übereinstimmt. Was haben Sie unternommen, um dies zu korrigieren? 3. Beschreiben Sie eine Situation, in der sich eine betroffene Persone nicht richtig ausdrücken oder ihren moralischen Standpunkt darstellen konnte. Wie haben Sie dazu beigetragen, die Meinung dieser Person zu «unterstützen» und ihr zu helfen, ihre Meinung in der Gruppe zu artikulieren? Wie haben Sie Ihre Neutralität angesichts der jeweiligen Wertvorstellungen aufrechterhalten?

Solche pädagogischen Schritte sind sachdienlich und notwendig. Die Schweizerische Akademie der Medizinischen Wissenschaften fordert in ihrer Empfehlung «Ethische Unterstützung in der Medizin» und bezüglich der grundsätzlichen Haltung von Mitgliedern einer Ethikstruktur u.a.: «Sie sind bereit, die eigenen Wertvorstellungen zu reflektieren. Sie sind bereit, aufgrund der gemeinsamen Diskussion eigene Ansichten zu überdenken. Sie sind offen für Gespräche und für Argumente, die von anderer Seite eingebracht werden. Sie sind offen für andere Disziplinen und Professionen. Sie sind bereit, ein realistisches Verständnis des klinischen Alltags zu erwerben.»

Allerdings erscheint eine in Übereinstimmung mit dem Vorstehenden zu treffende Umsetzung einer Auswahl- und Schulungspraxis für Mitglieder von Ethikkommissionen nicht gerade leicht (ohne an dieser Stelle von Bewertung / nachfolgender Supervision sprechen zu wollen). Fiester weiter: «Die nationalen bioethischen Organisationen warnten vor dem Risiko, dass Ethikberater eigene Werte aufoktroyieren könnten, doch die Problematik ist zu komplex, um durch einfache Richtlinien aus der Welt geschafft werden zu können.» Vielmehr bewegt man sich hier in einem Bereich, der sich durch Reglemente nur teilweise beeinflussen lässt und eher beruflichen, beziehungsspezifischen und deontologischen Haltungen zuzuordnen ist. Eine autoritätsfreie ethische Kultur lässt sich verstärkt durch Reflexion und vertieften interdisziplinären Austausch fördern. Die Autorin spricht von der «Fähigkeit des Zuhörens und des Bekundens von Interesse, Respekt, Unterstützung und Empathie». Dies sollten wir uns vor Augen halten - sowohl bei der Arbeit in den Ethikkommissionen als auch im praktischen Alltag. 